\title{
UNA MIRADA A LAS RELACIONES PARAVECINALES DE CHILE: LOS CASOS DE BRASIL Y ECUADOR (1990-2010)*
}

\section{A view into Chile's Non-contiguous Relations: the cases of Brazil and Ecuador (1990-2010)}

Irene Acevedo Albornoz ${ }^{* *}$

Abraham Quezada Vergara***

Jorge Riquelme Rivera ${ }^{* * * *}$

Recibido: 30 de octubre de 2012. Aprobado: 26 de febrero de 2013.

\section{RESUMEN}

Considerando que el tema de las relaciones paravecinales de Chile no ha sido profusamente analizado, concentrándose la literatura de manera predominante en las relaciones vecinales, el presente trabajo realiza una breve digresión teórica sobre el tema de la paravecindad, enfocándose en las relaciones de Chile con Brasil y Ecuador entre 1990 y 2010, en el marco de la prioridad regional declarada por la política exterior chilena. En tal sentido, se sostiene que las relaciones de Chile con ambos países mantienen matices. Por un lado, la marcada asimetría que influye, sin determinar, las relaciones chilenas con Brasil y, por otro, la relevante presencia de un actor vecinal catalizador en las relaciones con Ecuador.

PALABRAS CLAVE: Relaciones paravecinales, política exterior, Chile

* Los comentarios y opiniones efectuados por los autores son estrictamente a título personal y no comprometen, en modo alguno, al Ministerio de Relaciones Exteriores de Chile u otra entidad. El apartado referido a Ecuador se basa en los resultados de la investigación sobre las relaciones paravecinales chileno-ecuatorianas, que está siendo realizada por Abraham Quezada, en el marco de su tesis en el doctorado en estudios americanos de la Universidad de Santiago de Chile.

** Embajada de Chile en Brasil, Brasilia, Brasil. Correo electrónico: iacevedo@minrel.gov.cl

*** Embajada de Chile en Ecuador, Quito, Ecuador. Correo electrónico: aquezadav@minrel.gov.cl

${ }^{* * * *}$ Ministerio de Relaciones Exteriores de Chile, Santiago, Chile. Correo electrónico: jriquelme@minrel.gov.cl 


\begin{abstract}
Considering that the issue of Chile's relations with non-contiguous States has not been profusely analyzed, with literature concentrating mostly on neighbor relations, the present work takes a brief theoretic digression about the issue of non-contiguous relations, focusing on Chile's relations with Brazil and Ecuador from 1990 to 2010, in the context of the regional priorities established by Chilean foreign policy. In that sense, it is sustained that Chile's relations with both countries hold important distinctions. On one side, the marked asymmetry that influences, although does not determine, the relations between Chile and Brazil, and on the other, the relevant presence of an intermediary neighboractor in Chile's relations with Ecuador.
\end{abstract}

KEYWORDS: Non-contiguous relations, foreign policy, Chile

\title{
I. INTRODUCCIÓN
}

Las relaciones internacionales, como objeto de estudio, pueden definirse como todas aquellas acciones humanas, ya sean individuales o colectivas, que traspasan las fronteras de los tradicionales Estados-nación. Según Celestino del Arenal:

Las relaciones internacionales abarcan el conjunto de las relaciones sociales que configuran la sociedad internacional, tanto las de carácter político como las no políticas, sean económicas, culturales, humanitarias, religiosas, etc., tanto las que se producen entre los Estados como las que tienen lugar entre otros actores de la sociedad internacional y entre éstos y los Estados (2003:23).

Bajo este mismo enfoque multidimensional, Darío Batistella plantea que las relaciones internacionales corresponden al conjunto de las relaciones que tienen lugar más allá del espacio controlado por los Estados, cualquiera sea el actor -estatal o no- involucrado en estas relaciones, y cualquiera sea la naturaleza - política u otra- de estas relaciones (2003:27). Para los efectos de este trabajo, se examinarán particularmente las relaciones sostenidas a nivel estatal, concentrándose en las relaciones paravecinales, es decir, aquellas que tienen lugar entre países separados o intermediados por un actor estatal diferente. En este caso, se analizarán las relaciones de Chile con Brasil y Ecuador, a partir de 1990 hasta 2010. 
Cabe señalar que el análisis de las relaciones paravecinales de Chile no cuenta con una conceptualización ni una extensa bibliografía. Más bien, la literatura especializada es prácticamente inexistente, lo que puede definirse como un cierto problema de investigación. Los especialistas han preferido concentrarse en otras temáticas, como es el caso de las relaciones vecinales. Este trabajo pretende llenar en parte este vacío, así como superar este desafío metodológico.

Para ello, en este trabajo de carácter exploratorio, primero se contextualizará el estudio mediante un breve panorama de la política exterior de Chile hacia el entorno regional como marco de desenvolvimiento de las relaciones paravecinales chileno-brasileñas y chileno-ecuatorianas, respectivamente. Luego se esbozará una digresión teóricoconceptual de las relaciones paravecinales, para después enfocarse en las características y análisis concreto de las mencionadas relaciones bilaterales. Las conclusiones a las que se arribe en este trabajo se entregarán en el acápite final.

\section{LA POLÍTICA EXTERIOR DE CHILE HACIA EL ENTORNO REGIONAL COMO MARCO DE LAS RELACIONES PARAVECINALES}

Como política pública, la política exterior corresponde al conjunto de prioridades o preceptos establecidos por los líderes nacionales para servir como líneas de conducta a escoger, entre diversos cursos de acción (comportamientos), en situaciones específicas y dentro del contexto de su lucha por alcanzar sus metas (Pearson y Rochester 2004:113). En consecuencia, la política exterior es aquella política pública a través de la cual un país se inserta en el escenario internacional, considerando sus particulares intereses y objetivos nacionales. En última instancia, la política exterior está conformada por el conjunto de estrategias elegidas por un Estado para guiar su conducta en el ámbito internacional. Según sostiene Carlos Murillo: "La política exterior resulta de la interacción de diversos factores en múltiples ámbitos y niveles de acción, pero su carácter particular radica en que se convierte en la principal expresión de la conducta del Estado, en cuanto actor internacional" (2012:67).

Luego del aislamiento político que vivió Chile durante el régimen militar (19731990) por parte de un segmento importante de la comunidad internacional, desde el regreso a la democracia la política exterior de Chile ha buscado alcanzar la plena inserción internacional del país en el marco de un modelo de desarrollo basado en la apertura económica y comercial, de manera congruente con el principio del regionalismo abierto. Estas tendencias han sido coherentes con ciertos ejes fundamentales guiando la política 
exterior de Chile, como son la primacía de la democracia, el respeto y promoción de los derechos humanos y las libertades fundamentales, el fortalecimiento de las instancias de cooperación bilateral y regional, y una activa participación en las iniciativas políticas multilaterales, cuyo centro es la Organización de Naciones Unidas (Riquelme 2012:12021). Bajo la perspectiva de este trabajo, resulta especialmente relevante destacar que este proceso de reinserción buscó reposicionar al país como actor a nivel internacional, reinsertándolo en las corrientes globales con una perspectiva preferentemente regional.

Para Chile la prioridad por la región se constituye como uno de los elementos básicos de su política exterior, existiendo una especial preferencia por los países vecinos, fundamentalmente debido al enorme flujo de inversiones chilenas hacia estos, entre los que destacan Argentina y Perú como mayores receptores. Si bien esta dinámica no obedece a una estrategia planificada centralmente, por cuanto gran parte de los avances han sido efecto de inversiones privadas, de igual modo es posible apreciar que desde los gobiernos de la Concertación de Partidos por la Democracia las relaciones con los países del entorno vecinal y paravecinal resultaron una sección fundamental de la diplomacia chilena, por cuanto dichas interacciones se han transformado en un asunto de alta importancia en las vinculaciones del país.

La preeminencia de las relaciones vecinales y paravecinales también se debe, entre otros elementos, a que tales países forman parte integrante de los corredores bioceánicos, se constituyen como un origen de las diversas corrientes migratorias hacia el país, ${ }^{1} \mathrm{y}$ corresponden a socios potenciales para los proyectos de desarrollo compartido que el país requiere en áreas como la energía, la minería, el turismo o la infraestructura (Ross 2006).

También la integración regional ha estado en un lugar destacado de la política exterior, aunque a este respecto los énfasis han sido dispares, considerando el pragmatismo que históricamente ha seguido Chile en esta materia. Ejemplo de ello fue su preferencia de optar por la membresía como socio en el Mercado Común del Sur (Mercosur), antes que la calidad de miembro pleno, en virtud de las perspectivas ciertas de que esta última situación hubiera dañado la autonomía comercial del país. Además, se debe señalar la participación de Chile en la Comunidad Andina (CAN), a la que el país se incorporó en septiembre de 2006 en calidad de miembro asociado, así como su activa participación en la Unión de Naciones Suramericanas (Unasur), en la cual Chile ejerció la Secretaría Pro

1 A este respecto, se debe resaltar que de acuerdo a estimaciones del Departamento de Extranjería y Migración del Ministerio de Interior de Chile, 5,4 por ciento (19.089 personas) del total de la población extranjera residente en el país en 2009 (352.344 personas) es de origen ecuatoriano, mientras que 2,7 por ciento (9.624 personas) es brasileña (Departamento de Extranjería y Migración 2010:12). 
Témpore durante el 2009. Por último, cabe subrayar el liderazgo chileno en la conformación de la Alianza del Pacífico. Según el texto Perspectivas, proyecciones y desafíos de la política exterior de Chile, documento del Ministerio de Relaciones Exteriores de Chile, entre los intereses prioritarios de la política exterior está el "contribuir al fortalecimiento de la integración regional." En tal sentido, el texto sostiene que:

La integración de Chile a América Latina se ha constituido en uno de los elementos más importantes de nuestra política exterior, lo cual se refleja en el significativo número de proyectos que, en diferentes áreas, se orientan a acercarnos a los países de la región bajo un marco de un mutuo respeto y entendimiento (Ministerio de Relaciones Exteriores de Chile 2010a:21).

Luego, el documento plantea que el énfasis principal en esta línea está puesto en el desarrollo de la infraestructura regional, el fortalecimiento de la conectividad, la coordinación de políticas sociales, la reducción de la pobreza, la integración energética, el diálogo político y el libre comercio. En la misma línea, el excanciller Mariano Fernández ha sostenido: "La política exterior de Chile tiene como centro de gravedad la promoción de una identidad común en América Latina, región en la cual compartimos una historia, culturas, intereses y objetivos" (Fernández 2010:48).

Desde el retorno a la democracia, junto con reiterar los principios tradicionales de la política exterior chilena y reponer en la agenda la cooperación e integración regional -con estricto apego al desarrollo democrático y respeto a los derechos humanos-, los sucesivos gobiernos han privilegiado la inserción económica internacional a partir del modelo del regionalismo abierto, que se constituyó en uno de los pilares de la inserción positiva de Chile en el escenario global (Quezada 2010:121). El concepto del regionalismo abierto, nacido de la reflexión teórica de la Comisión Económica para América Latina y el Caribe de Naciones Unidas (Cepal) hacia comienzos de la década de 1990, en el marco de la denominada etapa neoestructuralista de dicha entidad regional, se refiere a las acciones que están orientadas a incrementar la interdependencia y la cooperación económica entre los países de una región, en el marco de una tendencia sostenida hacia el libre flujo de los factores productivos hacia el nivel global, traduciéndose, en el caso chileno, en una importante diversificación del destino de sus exportaciones en el curso de las dos últimas décadas.

Bajo el prisma del regionalismo abierto la política exterior de Chile busca insertar y proyectar comercialmente al país en distintas regiones y continentes del orbe. En la práctica, según destaca el ex director general de Política Exterior de Chile, embajador 
Carlos Portales, la apertura comercial diversificada del país en el marco del regionalismo abierto, ha implicado el logro paulatino de un mayor equilibrio entre las regiones de destino de los productos chilenos. Así, en 1990, Europa concentraba 51,7 por ciento de las exportaciones chilenas, Asia 21,4 por ciento, América del Norte 14,9 por ciento, América Latina y el Caribe 9,6 por ciento y otros mercados 2,5 por ciento; mientras, en el 2008, Asia recibió 37,3 por ciento de las exportaciones chilenas, Europa 26,1 por ciento, América del Norte (NAFTA) 16,9 por ciento, América Latina y el Caribe 16,1 por ciento y otros mercados 3,6 por ciento (Portales 2011:173-74). Esta diversificada apertura comercial ha servido para el país como una suerte de seguro ante los continuos vaivenes de la economía internacional.

El concepto de regionalismo abierto surgió en un momento en que casi todos los países de América Latina y el Caribe avanzaban hacia la apertura comercial y financiera, las privatizaciones y la reducción del papel del Estado como agente económico, en función de las ideas difundidas tras el Consenso de Washington. Para la Cepal, el regionalismo abierto corresponde a una:

reformulación del mensaje de integración regional presentada en los años cincuenta. Con el concepto de regionalismo abierto, la propuesta pasará a ser una combinación entre la apertura al resto del mundo y el incentivo a la integración regional, fórmula maximizadora de los beneficios de eficiencia mediante la inserción internacional, que conciliaba la apertura multilateral con la ampliación de la integración regional (Bielschowsky 2010:30).

En el marco del histórico pragmatismo de la política exterior de Chile, según sostiene Alberto van Klaveren (1998:126), esta idea acerca del regionalismo abierto se expresa de tres maneras fundamentales. Primero, las distintas opciones de inserción regional no son apreciadas como mutuamente excluyentes, sino que tienden a complementarse. Así, la inserción internacional múltiple del país implica que no existe una incompatibilidad entre las vinculaciones con América Latina, la Unión Europea y Asia. Segundo, los acuerdos están abiertos a la incorporación de nuevos miembros. Y tercero, la profundización de los esquemas regionales pretende hacerse compatible con la liberalización del comercio global, evitando, por lo tanto, el surgimiento de nuevas barreras en torno a los bienes y servicios que provienen desde fuera de la región.

Este nuevo regionalismo promueve políticas que vigoricen la integración, pero que al mismo tiempo sean compatibles con políticas que fortalezcan la competitividad internacional y el libre comercio. En suma, favorece la inserción internacional de la re- 
gión, minimizando los costos de esa inserción para los países. De tal modo, la estrategia chilena asume que la inserción internacional debe entenderse desde una perspectiva latinoamericana, considerando el tamaño relativo y la situación geográfica del país, así como las distintas experiencias de integración que se desarrollan en el mundo.

Junto con lo anterior, cabe señalar que la vinculación con la región obedece a una estrategia de inserción política y económica de carácter global, pues responde al interés chileno por fomentar un entorno regional estable para el comercio que permita, entre otros aspectos, plantear a Chile como un puente estratégico entre el océano Atlántico y el Pacífico, que vincule los mercados del Cono Sur y de Asia. Para ello, las inversiones en infraestructura e integración física resultan una cuestión fundamental (Aranda y Riquelme 2011:25).

Asimismo, la orientación hacia la región se explica por el interés chileno orientado a mejorar la manera en que se lleva a cabo la inserción internacional, en lo referido al avance hacia una segunda fase exportadora. Es decir, dentro de la plural y equilibrada inserción internacional chilena, estrechamente vinculada con su estrategia comercial, el país se ha orientado hacia los mercados de la región. En tanto el intercambio con los países del entorno implica el posicionamiento de productos chilenos de mayor valor agregado.

Cada uno de los elementos reseñados más arriba han estado presentes en las relaciones paravecinales del país, siendo los casos de Brasil y Ecuador especialmente representativos. Una vez realizada esta contextualización sumaria de las tendencias en la política exterior hacia el entorno regional, y antes de referirse a los casos concretos que aborda este artículo, cabe entonces realizar una breve exposición teórica sobre el análisis de las relaciones paravecinales de Chile. 


\section{LAS RELACIONES PARAVECINALES DESDE UNA PERSPECTIVA TEÓRICO-CONCEPTUAL}

Los estudios de política exterior de Chile en las últimas dos décadas si bien desde diversas perspectivas han reflexionado en torno a lo vecinal y lo regional, no han incluido la conceptualización de lo paravecinal como una categoría o ámbito distinto, con especificidades propias y como parte del espacio vecinal ampliado de Chile en la región (Quezada 2012:506).

Desde la década de 1990 en adelante, las relaciones internacionales de Chile en el espacio subregional se han desarrollado de forma continua y creciente, al producirse un nivel de interdependencia e interconexión mayor, consolidándose vínculos vecinales estables y crecientes. No obstante, en algunos casos esas aproximaciones se han visto tensionadas no solo por las sensibilidades crónicas de la llamada agenda histórica ${ }^{2}$, sino igualmente por los efectos no deseados que han acarreado los mayores niveles de interdependencia. Los flujos migratorios de ciudadanos, el incremento del comercio y los intercambios, por ejemplo, también han llevado una dosis de tráfico ilícito (productos y personas), disputas comerciales y conflictos variados. Asimismo, un factor adicional que ha contribuido a generar una cierta animadversión hacia Chile en su entorno vecinal ha estado en la irritación que ha provocado la imagen de país exitoso o de país referente, que proyecta hacia dicho espacio.

Durante este período, la anterior situación requirió extremar los contactos interestatales para la coordinación de respuestas eficientes a los nuevos desafíos y a las problemáticas suscitadas. Si bien esa dinámica también se dio en el ámbito paravecinal, comparativamente fue en menor intensidad que la desarrollada en el plano vecinal, no alcanzando por lo tanto a impactar la agenda de relaciones entre dichos países. Así, este tipo de aproximación practicada abarcó una variada gama de actividad bilateral, como las vinculaciones políticas, el intercambio comercial, cultural y la cooperación en un sentido amplio, incluyendo materias de defensa, las de mayor desarrollo.

Más allá de la comprensión de lo paravecinal desde el punto de vista puramente instrumental y descriptivo, y tratando de visualizarlo de manera sustantiva y con algún valor analítico comparativo, es pertinente una pregunta esencial: ¿qué se dice cuando se habla de lo paravecinal? Desde el punto de vista etimológico, el prefijo griego para significa "junto a", por lo que sería aquello que se encuentra o localiza inmediato a lo vecinal.

2 Entendida como un conjunto de prejuicios, desconfianzas y resquemores mantenidos por los países en el marco vecinal, derivados de antiguos conflictos y enfrentamientos o de la percepción de actitudes hostiles entre ellos. 
Otra de las acepciones consideradas corresponde a la preposición para, la cual sugiere la idea de relación de una cosa con otra. Este último significado le agrega una dimensión relacional importante que permite avanzar en su esclarecimiento.

De este modo, la idea de la proximidad territorial no inmediata está acompañada de la dimensión de lo relacional, que es el eje central en la política internacional en una gran variedad de aspectos. Ambos elementos conviven en la conceptualización de lo paravecinal no solo desde el punto de vista semántico, sino también desde el ámbito concreto de la práctica de las relaciones internacionales.

Los países paravecinales, al estar separados o intermediados por uno o varios actores estatales diferentes, pueden desarrollar sus nexos en estrecha relación con lo vecinal. Así, el significado de lo paravecinal, junto con dar cuenta de una variable sistémica, está necesariamente subordinado a la idea de lo vecinal, lo cual, como concepto-eje, puede nuclear e intermediar la relación entre esos países. Como se verá, este ha sido el caso de las relaciones chileno-ecuatorianas, aunque respecto de las relaciones chileno-brasileñas la situación ha sido distinta.

Una enunciación todavía provisional y en desarrollo, indica que lo paravecinal debería identificarse con un ámbito espacial o geográfico ubicado más allá del entorno colindante inmediato y sobre el cual el país ejerce acciones de política exterior o mantiene vínculos transnacionales, que pueden tener su origen en situaciones que lo enlazan con el Estado contiguo geográficamente. Este rango de interacciones, por tanto, puede ir desde la simple paravecindad geográfica, pasando por estrechos nexos comerciales, estratégicos o migratorios, hasta constatar la existencia de una relación de trilateralidad (Stallings y Székelyet 1993) o de vasos comunicantes en donde, en definitiva, las políticas y acciones respecto del Estado paralimítrofe están determinadas, en alguna medida, por la relación vecinal inmediata y/o por la presencia/ausencia de actores no estatales que empujan dicho proceso.

Lo paravecinal, para el caso de Chile, aparece o se configura al trilateralizarse el escenario subregional entre países que mantengan entre sí recelos, diferencias o conflictos relacionados con la denominada agenda histórica. La respuesta de la política exterior debería expresarse, en consecuencia, en acciones necesarias para contrarrestar o detener las pretensiones del Estado intermedio, que potencialmente podrían afectar el interés nacional de alguno de los actores laterales. Así se comprueba que la importancia de Brasil y Ecuador en los equilibrios que a Chile le han interesado en la región ha sido esencial y, de algún modo, irreemplazable. El país se ha mantenido atento frente a la ocurrencia 
de circunstancias que, aun sin proponérselo, puedan debilitar la presencia y la natural influencia que el país ejerce en el espacio regional sudamericano, especialmente en la vertiente del Pacífico sudeste y en el área andina.

\section{LAS RELACIONES CHILE-BRASIL}

Brasil y Chile comparten los valores democráticos fundamentales y coinciden en potenciar los esfuerzos por llevar adelante la concertación política, la integración regional y el fortalecimiento de las instituciones internacionales multilaterales. En este contexto, la relación política de ambos países se ha desarrollado mediante la creación de mecanismos de vinculación que funcionan en forma periódica, como la Comisión Bilateral, que es el principal mecanismo de diálogo y cooperación que se reúne a nivel de ministros de Relaciones Exteriores. También se estableció el mecanismo de consultas políticas que opera a nivel de directores generales de los Ministerios de Relaciones Exteriores, que se ocupa de la globalidad de la relación bilateral y permite analizar en profundidad las materias de interés común. Simultáneamente, se desarrollan encuentros en los ámbitos de la defensa, consultas entre autoridades aeronáuticas y a nivel parlamentario.

En relación con la integración física se estableció una Comisión Técnica Bilateral Chile-Brasil, que aborda materias relativas a la conectividad de ambas naciones, mediante el análisis de temas referentes a transporte, aduanas e infraestructura. El Acta de Instalación de la Comisión Bilateral Chile-Brasil, suscrita el 11 de febrero de 2010 en Santiago, establece en su artículo tercero los mecanismos de diálogo bilateral, las comisiones mixtas y los grupos de trabajo, que se mencionan en el presente estudio.

A pesar de las diferencias geográficas, políticas y económicas, Chile y Brasil han tenido, a lo largo de su historia, una relación bilateral que se ha caracterizado por el encuentro y el trabajo conjunto. Ambos países han participado en acciones conjuntas en el plano internacional -como es el caso de la activa labor en la Misión de Estabilización de Naciones Unidas en Haití (MINUSTAH) y en el Consejo de Defensa Suramericano- que ha contribuido a incrementar y consolidar la confianza mutua y buscar complementar sus intereses, dejando de lado cualquier dinámica de competencia. Para Chile, su condición paravecinal con Brasil lo sitúa en una posición cómoda y hasta beneficiosa, al no tener en su agenda el tema limítrofe que genere ruido en sus continuos procesos negociadores de acuerdos. Brasil ha sido un aliado histórico de Chile en momentos en que este último ha tenido diferencias con sus vecinos. 
Los primeros contactos entre el Imperio del Brasil y Chile datan del 1 de septiembre de 1838, fecha en la cual se suscribió un Tratado de Amistad, Comercio y Navegación entre ambos países. Brasil tiene un territorio, población y riqueza de recursos naturales que le favorecen ostentar un desarrollo económico sostenido y de gran proyección mundial. Ante este panorama, Chile, que no cuenta con indicadores geográficos de la dimensión brasileña, se relaciona con dicho país mediante el desarrollo de una economía estable, una solidez política y un capital intangible de prestigio que le permiten jugar un rol de puente entre esta gran economía y el mundo en desarrollo.

En este marco general, a finales de las décadas de los 80 y comienzos de los 90 del siglo pasado, Chile y Brasil experimentaron una coincidencia temporal de recuperación de la democracia, donde se creó un clima favorable para la concertación de posiciones y la cooperación en distintos niveles y áreas temáticas. La prioridad de la política exterior de Brasil fue la readecuación de su agenda económica internacional, a la vez de lograr la reinserción del país en el mundo, una vez superados los tiempos de la dictadura. En este marco, el impulso al Mercosur constituyó el eje central de la integración regional (Acevedo 2011). En cambio, Chile puso su énfasis en una pragmática reinserción internacional, bajo los preceptos del regionalismo abierto, en tanto había heredado del régimen militar la modalidad de posicionamiento en las corrientes del capitalismo global.

A nivel político, en los últimos 20 años la relación bilateral entre Chile y Brasil ha sido dinámica y fluida, a pesar de que los encuentros presidenciales se realizan de manera más frecuente en reuniones cumbres que en visitas oficiales bilaterales. A nivel de cancilleres, los encuentros periódicos se formalizaron en 2010, fecha en que se instaló la Comisión Bilateral Chile-Brasil. Entre los temas de mayor relevancia que se han desarrollado, se encuentran la televisión digital y la creación de grupos de trabajo de cooperación en materia de salud, desarrollo social e integración productiva.

Cabe destacar que los encuentros a nivel de cancilleres generan una comunicación de primer nivel en apoyo a los procesos de negociación que se llevan a cabo para intensificar la relación política y comercial. Asimismo, mediante el Acta de Instalación de la Comisión Bilateral Chile-Brasil, se estableció el mecanismo de consultas parlamentarias, que permite el diálogo entre las Comisiones de Relaciones Exteriores de los Senados de ambos países. Dicha instancia permanente de consulta se reúne trienalmente, de manera alternada en ambos países, ampliando el espectro de la relación bilateral hacia otros poderes de los Estados. 
En el terreno económico, se debe subrayar que la relación entre Chile y Brasil se rige en gran parte mediante las regulaciones establecidas en el Mercosur, del cual Brasil es miembro pleno y Chile un país asociado. Cada elemento de la relación por tanto, debe estar en la línea de lo establecido por el Acuerdo de Complementación Económica $\mathrm{N}^{\circ} 35$ Mercosur-Chile (1996), que funciona como un referente marco para la relación de ambos países. El siguiente cuadro expresa la evolución del comercio entre ambos países, durante el ciclo 2006-2010:

\section{Cuadro No 1}

Evolución del intercambio comercial Chile-Brasil años 2006-2010

\begin{tabular}{|c|c|c|c|c|c|}
\hline Millones US\$ & 2006 & 2007 & 2008 & 2009 & 2010 \\
\hline Exportaciones & 2.837 & 3.391 & 3.861 & 2.736 & 4.213 \\
\hline Importaciones & 4.281 & 4.529 & 5.301 & 2.880 & 4.659 \\
\hline Balanza Comercial & -1.160 & -852 & -1.082 & 37 & -199 \\
\hline Intercambio Comercial & 7.118 & 7.920 & 9.222 & 5.616 & 8.872 \\
\hline
\end{tabular}

Fuente: Embajada de Chile en Brasil (s/f).

De acuerdo a un informe de la Dirección de Relaciones Económicas del Ministerio de Relaciones Exteriores de Chile, el total de las exportaciones chilenas a Brasil constituyen a este último como el sexto socio comercial del país austral (Ministerio de Relaciones Exteriores de Chile 2011). Asimismo, en materia de inversiones, Brasil es el segundo país de destino de las inversiones directas de capitales chilenos en el mundo, después de Argentina, con un monto de US\$ 1.177 millones en 2010. De esta cifra 73 por ciento fue destinado a proyectos en el sector industrial, le siguen el sector servicio y energía. Brasil es un país muy atractivo para la inversión chilena, lo que se ha demostrado por el creciente flujo de capitales colocados en dicho país en las últimas décadas. Esta inversión se destaca por su capacidad generadora de empleo, lo que contribuye a crear una positiva percepción de la ciudadanía brasileña. La inversión de Chile en Brasil y otros países es de carácter horizontal, es decir, se continúa en el extranjero lo que se hace bien en el interior del país. Respecto del sector servicios, destacan el transporte aéreo y marítimo, el retail y las tecnologías de la información.

Las inversiones de Brasil en Chile se destacan por la preferencia en el sector minería, seguido por los servicios financieros. De acuerdo a lo informado por el Comité de Inversión Extranjera de Chile, la inversión directa proveniente de Brasil acumuló US\$ 798,1 millones en 2010. La inversión brasileña en Chile representa un porcentaje bastante menor del total de sus inversiones en el mundo, con 0,8 por ciento de la inversión directa efectiva. Actualmente, la agenda económica ha estado centrada en la liberalización de 
comercio de servicios, donde se espera materializar un protocolo de servicios a las legislaciones nacionales, armonización de estadísticas de comercio bilateral y el análisis de factibilidad de negociar en materia de compras públicas.

En la relación comercial, uno de los mecanismos de mayor importancia es la Comisión de Comercio Bilateral, que tiene por objetivo fomentar el diálogo en materia de comercio bilateral para permitir la resolución de materias particulares en dicho ámbito, promoviendo, asimismo, la creación de comercio bilateral, mediante estrategias conjuntas de mediano y largo plazo, e incentivando y facilitando el desarrollo de contactos directos entre los distintos organismos públicos y privados.

Una de las áreas que Brasil ha demostrado interés en profundizar en la relación económica con Chile, es el área agrícola. Desde 2007 se desarrollan temas a nivel ministerial, con los términos de referencia, modalidad y agenda de trabajo. Las materias de interés para Brasil son la cooperación técnica y asuntos sanitarios y fitosanitarios, y para Chile la investigación, cooperación técnica y agricultura familiar campesina y temas sanitarios y comerciales.

En lo relativo al ámbito político-estratégico, cabe destacar el Acuerdo de Cooperación en materia de defensa suscrito por ambos países en 2007, que incluye la promoción de la cooperación, con énfasis en las áreas de investigación y desarrollo, apoyo logístico, adquisición y obtención de equipamiento y servicios de defensa. También contempla el intercambio de conocimientos y experiencias operativas y de ejercicios, y la utilización de equipamiento militar en la capacitación en el área de operaciones de paz. Además, incluye cooperación en las áreas de ciencia y tecnología, en la promoción de actividades combinadas de instrucción, entrenamiento y ejercicios, en el intercambio de conocimientos y experiencias en la utilización de equipamiento militar, nacional y extranjero, y en la promoción del intercambio académico del ámbito de defensa.

Por último, es relevante subrayar la vinculación que tienen ambos países en el tema antártico. Brasil es uno de los principales usuarios del Aeródromo Teniente Marsh ubicado en la isla Rey Jorge. Brasil donó en el 2009 un sistema de navegación avanzada y posicionamiento para dicho aeródromo, el cual permitirá disminuir la cancelación de vuelos programados y la cantidad de aterrizajes frustrados, fortaleciendo el objetivo de consolidar a Chile como país puente a la Antártida. Si bien Brasil adhirió al Tratado Antártico en 1975 (sin efectuar reclamación territorial), su participación en él se realiza de forma menos activa que Chile y otros países de la región. A este respecto, la relación 
bilateral entre ambos países se caracteriza por una estrecha cooperación en los ámbitos antárticos logístico y científico.

En este tema, ambos países se conectan con la tendencia a nivel mundial de asociar la Antártida al desarrollo de la ciencia, la tecnología y la innovación, en un marco de cooperación internacional. Chile es modelo para Brasil en el diseño de su política científica antártica. Esto se debe al fuerte impulso que ese país está dando a la ciencia en dicho continente, siguiendo lo realizado por el Instituto Antártico Chileno que ha asesorado, en el diseño, al Programa Antártico Brasileño.

Chile y Brasil manifiestan diferencias relevantes en sus indicadores geográficos, la presencia de riquezas naturales y su capital humano. Ello influye en la gravitación y jerarquía que se le asigna al otro por parte de cada nación, en su relación política bilateral. Como se aprecia en los acuerdos y temáticas desarrolladas, para Chile resulta absolutamente beneficioso contar con un aliado de estas dimensiones, ya que le permite expandir su comercio exterior hacia un país con un mercado y potencialidades de dimensiones continentales.

La proximidad geográfica, sin ser limítrofe, favorece un acercamiento con altos niveles de complementariedad, ya que Chile se presenta como país aliado estratégico para Brasil en cuestiones políticas y comerciales. Estas consideraciones resultan fundamentales a la hora de entender las tendencias que ha seguido la política exterior chilena hacia el gigante sudamericano. El trabajo conjunto y coordinado entre ambas naciones se expresa de manera destacada en la ayuda para la crisis y posterior reconstrucción en Haití y en las posiciones comunes que ambos países sostienen en foros y organismos de carácter multilateral, como es el caso de Naciones Unidas y la Organización Mundial del Comercio (OMC), según sostuvo el canciller brasileño Celso Amorim en Santiago el 2010 (Ministerio de Relaciones Exteriores 2010b). Asimismo, cabe señalar que Chile otorgó un apoyo unilateral a la aspiración de Brasil de ingresar -como miembro permanente- en el Consejo de Seguridad de Naciones Unidas. En suma, para el país andino, Brasil, que se encuentra entre las 10 primeras economías del mundo, ha demostrado en los últimos años tener un amplio espacio para impulsar y desarrollar el mutuo crecimiento y desarrollo en un variado espectro de actividades. 


\section{LAS RELACIONES CHILE-ECUADOR}

Las vinculaciones bilaterales entre Chile y Ecuador a lo largo de su historia dan cuenta de una gran cercanía en múltiples áreas, destacándose los aspectos y coincidencias desde lo cultural e idiomático, pasando por la consolidación republicana y territorial y el activismo externo, hasta el hecho de estar ambos países expuestos a la influencia de similares fenómenos regionales y globales.

Desde la consolidación del proceso de independencia y a partir de la guerra contra la Confederación Perú-Boliviana, Chile comprendió la importancia que podían jugar actores paravecinales como Colombia y Ecuador en ese conflicto. Así, en 1837 envió a Quito una misión a cargo de Ventura Lavalle no solo por el peligro objetivo que las ambiciones del mariscal Santa Cruz revestían para "la independencia de Bolivia y Ecuador", sino porque se consideraba como "absolutamente necesaria para la seguridad de los otros Estados sudamericanos" (Burr 1974:40). Una variante política-comercial de ese acercamiento con Ecuador se apreció en 1856, cuando Chile suscribió el Tratado Continental o Tripartito con ese país. De este modo, un factor importante en el origen de la aproximación paravecinal de Chile y Ecuador, especialmente luego de la Guerra del Pacífico, y contrariamente a lo apreciado en las relaciones chileno-brasileñas, han estado históricamente catalizadas por la presencia vecinal de Perú.

$\mathrm{Al}$ adquirir Chile una posición dominante luego del conflicto bélico con Perú y Bolivia, lo paravecinal cobró renovada importancia. Las sucesivas colaboraciones con Ecuador a través de la modalidad del envío de misiones militares practicadas por Santiago, tuvieron por propósito mantener un estrecho contacto con un aliado en caso de conflicto con algún país vecinal, situación que Ecuador a su vez aprovechó para enfrentar y fortalecer su alicaída posición en materia limítrofe, que padeció con sus vecinos Perú y Colombia.

Desde 1990 se aprecia una continuidad en las vinculaciones entre ambos países, que se expresa en una importante cuota de reciprocidad de visitas, incluyendo la presencia de primeros mandatarios, altas autoridades, oficiales de las Fuerzas Armadas y otras. Las consultas políticas se han validado como un instrumento eficaz para estructurar y ampliar los nexos bilaterales en sus diferentes áreas, aunque las realidades internas de cada país han estado determinadas por la dicotomía estabilidad, crecimiento y paz vecinal exhibidos por Chile versus inestabilidad, crisis y enfrentamiento bélico experimentados por Ecuador. En medio de las decisiones basadas en los criterios del regionalismo abierto, la diplomacia chilena ha favorecido la aparición de una clara modalidad paravecinal de 
inserción, al participar Chile activamente como garante en el conflicto del Cenepa de 1995 y su posterior proceso de paz, sin descuidar por ello las otras áreas de la relación.

En el ámbito comercial, se constata que el intercambio entre Chile y Ecuador es significativamente menor respecto del de Brasil. El siguiente cuadro expresa la evolución del intercambio entre Chile y Ecuador, durante el período 2006-2010:

\section{Cuadro No 2}

Evolución del intercambio comercial Chile-Ecuador entre 2006 y 2010

\begin{tabular}{|c|c|c|c|c|c|}
\hline Millones US\$ & 2006 & 2007 & 2008 & 2009 & 2010 \\
\hline Exportaciones & 434 & 460 & 512 & 420 & 474 \\
\hline Importaciones & 577 & 717 & 1.530 & 894 & 765 \\
\hline Balanza comercial & -143 & -257 & -1.019 & -474 & -292 \\
\hline Intercambio comercial & 1.01 & 1.177 & 2.040 & 1.314 & 1.239 \\
\hline
\end{tabular}

Fuente: Embajada de Chile en Ecuador (s/f).

En un nivel general, cabe anotar que, según informes de la Dirección de Relaciones Económicas del Ministerio de Relaciones Exteriores de Chile, el intercambio comercial entre ambos países saltó desde los US\$ 121 millones, en 1990, a los US\$ 338 millones, en 1999, para llegar en el 2010 a los US\$ 1.239 millones. No obstante, los niveles de inversión se han mantenido bajos en monto. Entre otras razones, como consecuencia de la poca seguridad jurídica que ofrecía Ecuador. De todos modos, y pese a los episodios de conflictividad y crisis que ha padecido este país, la relación se ha consolidado en el ámbito político, económico y cultural, así como en lo relativo a la cooperación en el ámbito defensivo-estratégico.

Mientras Chile profundizó su inserción en la economía mundial, su imagen-país a fines de los gobiernos de la Concertación de Partidos por la Democracia se consolidó como un referente significativo para Ecuador no solo como un modelo a seguir en materia de ejercicio democrático o de sana y adecuada gestión económica, sino en otra multiplicidad de áreas en las cuales Ecuador solicitó y obtuvo cooperación en ese período. Invariablemente en estos años el nombre del país en el espacio mediático ecuatoriano estuvo asociado a las virtudes enunciadas precedentemente.

Desde el punto de vista de la aproximación paravecinal de Chile hacia Ecuador desde 1990, se observan ciertos matices que cabe enunciar. En los años 90, como país garante en el conflicto del Cenepa (1995-1998), Chile actuó con imparcialidad en la disputa entre Perú y Ecuador, pero con elementos de una neutralidad activa, tal como había 
ocurrido en la misma controversia de 1941-1942, que condujo a la firma del Protocolo de Río entre los mismos países. Posteriormente se conocieron denuncias de venta de armas chilenas a Ecuador durante el conflicto del Cenepa, las cuales Santiago rechazó, argumentando que los envíos denunciados fueron despachados antes del inicio de esas hostilidades. Si bien en lo formal el papel de garante implicó equidistancia, ello no impidió la profundización paravecinal cada vez que ello se estimó necesario. En la década del 2000, las relaciones con Ecuador se fundamentan en la emergencia de las incidencias asociadas al límite marítimo planteado por Perú, lo cual determinó que este fuera un período profundamente paravecinal.

Sin importar los momentos de precaria estabilidad institucional y de inestabilidad política y crisis de gobernabilidad, que para el caso ecuatoriano ocurrieron entre 1979 y hasta el 2006, Santiago, a partir de sus preocupaciones paravecinales, ha desarrollado y mantenido una política de vinculaciones activas hacia el país andino, fortaleciendo el diálogo político y la generación de nuevas instancias para canalizar la cooperación y la integración, convergiendo esfuerzos políticos y diplomáticos, que han dado cuenta de esa preocupación hacia el área andina y pacífica.

Por ello, Chile objetivamente ha buscado acercarse a Ecuador, entre otras cosas, para ayudar a cambiar el escenario de inestabilidad y crisis del país andino, debido a que, como señaló el ex canciller ecuatoriano José Ayala Lasso, "a nadie le interesa tener un socio estratégico que sea pobre, atrasado, inculto y con altos grados de inestabilidad, que dificulta consolidar sus relaciones comerciales" (entrevista personal, 8 de enero de 2012).

Los esfuerzos realizados por Chile se han materializado en diversos logros bilaterales, como el acuerdo para la creación del Consejo Interministerial Binacional (2007) y el Acuerdo de Asociación (2008), a lo cual se unió el correspondiente Acuerdo de Complementación Económica No 32 y No 65 (1994 y 2008), y los acuerdos de Protección Recíproca de Inversiones (1993) y el Convenio para Evitar la Doble Tributación (1999), aunque este último solo entró en vigencia en el 2004. A lo anterior se agrega una gran cantidad de convenios interinstitucionales e interministeriales, sumatoria diplomáticoeconómica que en el mismo período no se da o se aprecia solo varcialmente en las relaciones de Chile con otros actores de la región.

Un rubro relevante en esa aproximación paravecinal ha estado en la cooperación en un sentido amplio, la cual se evidencia en una multiplicidad de acuerdos, programas de trabajo y en acciones de ayuda y asistencia. Actualmente, la Agencia de Cooperación Internacional de Chile (AGCI) promueve diversos aportes hacia Ecuador, en materias 
tales como formación de recursos humanos y becas, asistencia técnica y en cooperación triangular. En una visión de conjunto, de los US\$ 7,6 millones otorgados por la Agencia de Cooperación Internacional de Chile (AGCI) en la década que va de 1999 al 2009 al conjunto de los siete países vecinales y paravecinales, la prioridad vecinal de la cooperación chilena estuvo radicada en Bolivia y Perú, pero en la paravecinal prevaleció Ecuador (Agencia de Cooperación Internacional de Chile 2010).

Ello se debe, sin duda, a las demandas en Ecuador, las cuales han sido abordadas vía cooperación y a la existencia de la Agencia de Cooperación Internacional de Chile (AGCI) y sus contrapartes ecuatorianas, que contemplan los planes, estrategias e instrumentos necesarios para el fomento de la cooperación y la identificación de las fuentes para la obtención de recursos. También en ello ha estado la racionalidad política de Santiago, respecto de la necesidad de fortalecer lazos con el país paravecinal, especialmente desde que se conoció la decisión peruana de demandar a Chile en la Corte Internacional de Justicia por la delimitación marítima.

Fuera del contexto de la Agencia de Cooperación Internacional de Chile (AGCI), la cooperación entre ambos países actualmente se expresa igualmente en ámbitos relevantes, como el minero y el energético, donde destaca el acuerdo suscrito entre la Empresa Nacional del Petróleo de Chile (ENAP) y PetroEcuador (octubre de 2008). Asimismo, en septiembre de 2008 y en junio de 2009, se suscribieron convenios entre los ministerios del ramo para emprendimientos conjuntos. Otras áreas de cooperación se han dado en el campo de la defensa y en gestión policial, como ha sido el trabajo conjunto entre Chile y Ecuador en el marco de la MINUSTAH y de desminado humanitario a través de Marminas-OEA, desde el 2005 en adelante, espacio de cooperación en donde Brasil igual desempeña un papel importante.

\section{CONCLUSIONES}

En este trabajo se definieron las relaciones paravecinales como aquellas que se llevan a cabo entre países separados o intermediados por un actor estatal diferente. Para Chile, estas relaciones deben entenderse en el marco de la prioridad por la región declarada en el contexto de su política exterior.

Respecto de las relaciones chileno-brasileñas en particular, se planteó que ambos países comparten valores políticos fundamentales, como la defensa y promoción de la democracia y, en el ámbito internacional, el impulso de la integración regional y el mul- 
tilateralismo. Si bien las relaciones podrían caracterizarse como asimétricas, debido a la dimensión territorial, política y económica del coloso sudamericano, lo cierto es que las relaciones entre Brasil y Chile pueden calificarse, en términos generales, como fluidas y dinámicas. La enorme dimensión relativa de Brasil en distintas esferas tiene como contraparte la imagen de Chile como un país estable política y económicamente y con prestigio en el terreno de las instituciones multilaterales, como un país puente entre el mundo desarrollado y el mundo en desarrollo.

Miradas en el largo plazo, las sólidas relaciones entre ambos países se expresan en un relevante intercambio comercial, que se rige por el Acuerdo de Complementación Económica $\mathrm{N}^{\circ} 35$ de 1996, cooperación en la esfera de la defensa y en un activo intercambio político en el ámbito internacional. A este último aspecto, cabe destacar el apoyo de Chile para que Brasil ocupe un asiento permanente en el Consejo de Seguridad de Naciones Unidas y el trabajo conjunto en el contexto de la Misión de Estabilización de las Naciones Unidas en Haití (MINUSTAH)

Por su parte, las relaciones chileno-ecuatorianas deben entenderse bajo una perspectiva histórica y geopolítica. Las vinculaciones entre estos países dan cuenta de una importante cercanía en múltiples áreas, tales como el ámbito político, el intercambio comercial, lo cultural y la cooperación internacional, así como por el hecho de estar ambos Estados influenciados por similares fenómenos regionales y globales. Sin embargo, el factor de mayor relevancia que incide en esta relación paravecinal, a partir de la Guerra contra la Confederación Perú-Boliviana y, sobre todo, luego de la Guerra del Pacífico -contrariamente a lo apreciado en las relaciones chileno-brasileñas-, es la presencia del vecino común -Perú-, el cual en relación a ambos ha manifestado ciertas problemáticas: con Ecuador indefiniciones limítrofes y enfrentamientos en el siglo XX y con Chile demandas por el límite marítimo.

En este marco general, las relaciones entre Chile y Ecuador desde 1990 están marcadas por la continuidad, lo que se expresa en periódicas y recíprocas visitas oficiales, intercambio económico, migración y cooperación en variados ámbitos, incluido el defensivo, aunque cabe señalar que las relaciones han estado igualmente mediatizadas por las realidades internas de cada país. En este sentido, se aprecia una dicotomía entre la estabilidad, crecimiento y paz vecinal exhibidos por Chile y la inestabilidad, crisis y enfrentamiento bélico experimentados por Ecuador.

En consecuencia, ambas relaciones de carácter paravecinal, junto con formar parte importante de la política exterior de Chile, presentan características distintas. Por un 
lado, la marcada asimetría que influye, aunque no determina, las relaciones chilenas con Brasil y, por otro, la influyente presencia determinante de un actor vecinal catalizador en las relaciones con Ecuador. En ambos casos no existe una abundante teorización ni bibliografía, y menos aún bajo la perspectiva de las relaciones paravecinales de Chile. Con este trabajo se espera, al menos en parte, haber contribuido a llenar este vacío.

\section{REFERENCIAS}

1. Acevedo, Irene. 2011. "Perspectivas de la política exterior de Brasil en Chile y Argentina: posiciones nacionales ante la reforma del Consejo de Seguridad." Pp. 613-21 en Miradas Transcordilleranas: Selección de trabajos del IX Congreso Argentino-Chileno de Estudios Históricos e Integración Cultural, compilado por P. Núñez. San Carlos de Bariloche: Universidad Nacional de Río Negro.

2. Agencia de Cooperación Internacional de Chile. 2010. Información estadística sobre cooperación otorgada por AGCI a países vecinales y paravecinales desde 1999 a 2009 (inédito). Santiago: Departamento de Política y Planificación.

3. Aranda, Gilberto y Jorge Riquelme. 2011. "La política exterior de Chile desde 1990. Inserción internacional y prioridad regional." Cuadernos sobre Relaciones Internacionales, Regionalismo y Desarrollo 10:11-41.

4. Arenal, Celestino del. 2003. Introducción a las relaciones internacionales. Madrid: Tecnos.

5. Ayala, José. 2012. Entrevista personal, realizada por Abraham Quezada. 8 de enero.

6. Batistella, Darío. 2003. Théorie des relations internationales. Bordeaux: Presses de Sciences Po.

7. Bielschowski, Ricardo, comp. 2010. Sesenta años de la Cepal. Textos seleccionados del decenio 1998-2008. Buenos Aires: Cepal-Siglo Veintiuno Editores.

8. Burr, Robert. 1974. By Reason or force: Chile and the balancing of power in South America, 1830-1905. Berkeley: University of California Press.

9. Departamento de Extranjería y Migración. 2010. Informe Anual. Santiago de Chile: Ministerio del Interior.

10. Embajada de Chile en Brasil. S/f. Comercio Exterior. Consultado el 6 de febrero de 2013. (http://chileabroad.gov.cl/brasil/asuntos-comerciales/oficina-comercial/comercio-exterior/) 
11. Fernández, Mariano. 2010. “América Latina: Su integración, prioridad en la política exterior de Chile." Pp. 48-57. El mundo desde el sur. Voces de Chile en política exterior, editado por el Ministerio de Relaciones Exteriores de Chile. Santiago de Chile: Gráfica Puerto Madero.

12. Ministerio de Relaciones Exteriores de Chile. 2010a. Perspectivas, proyecciones y desafíos de la política exterior de Chile. Santiago de Chile: Maval Impresores.

13.__ 2010b. Comunicado "Cancilleres de Chile y Brasil instalan Comisión Bilateral." Consultado 20 de octubre 2012. (http://www.minrel.gob.cl/prontus_minrel/site/artic/20100211/ pags/20100211211049.php)

14_-2011. "Evaluación de las relaciones comerciales entre Chile y el Mercosur a quince años de la entrada en vigencia del Acuerdo de Complementación Económica." Consultado 20 de octubre 2012. (http://www.direcon.gob.cl/sites/www.direcon.gob.cl/files/bibliotecas/ bibliotecas_11_11_11164955.pdf)

15. Murillo, Carlos. 2012. "Política exterior: una política pública sui generis." Revista Peruana de Ciencia Política 1:55-90.

16. Pearson, Frederic y Martin Rochester. 2004. Relaciones Internacionales, situación global en el siglo XXI. Bogotá: Mac Graw Hill.

17. Portales, Carlos. 2011. "Desafíos para la política exterior en las próximas décadas: Chile y la región en un mundo global.” Estudios Internacionales 169:171-93.

18. Quezada, Abraham. 2010. "Inserción internacional de Chile en la post-Guerra Fría. Concertación política e integración económico-comercial; dos ejes conceptuales de la política exterior en el Gobierno de Ricardo Lagos (2000-2006).” Enfoques 13:119-34.

19. - 2012. "Relaciones de Chile con Centroamérica y el Caribe; pasado, presente y los nuevos desafíos." Pp. 503-28 en La política exterior de Chile, 1990-2010, del aislamiento a la integración global, editado por M. Artaza y C. Ross. Santiago de Chile: RIL Editores.

20. Riquelme, Jorge. 2012. "La política exterior de Chile y la Unión Europea. A diez años del Acuerdo de Asociación.” Relaciones Internacionales 42:119-51.

21. Ross, César. 2006. “Chile: los desafíos de la política exterior de Michelle Bachelet." Foreign Affairs en Español. Abril-junio. Consultado 29 de octubre 2012. (http://www.internacionaldelconocimiento.org/documentos/articulos_publicaciones/Z1.pdf) 
22. Stallings, Bárbara y Gabriel Székelyet, eds. 1993. Japón, los Estados Unidos y la América Latina. ¿Hacia una relación trilateral en el hemisferio occidental? México: Fondo de Cultura Económica.

23. Van Klaveren, Alberto. 1998. "Inserción internacional de Chile.” Pp. 117-60 en Chile en los noventa, editado por C. Tolosa y E. Lahera. Santiago: Presidencia de la República. 